\title{
Selective compounds enhance osteoblastic activity by targeting HECT domain of ubiquitin ligase Smurf1
}

\author{
Yuan Zhang ${ }^{1}$, Cheng Wang ${ }^{1,2}$, Yu Cao ${ }^{1,3}$, Yongqing Gu ${ }^{1,2}$ and Lingqiang Zhang ${ }^{1}$ \\ ${ }^{1}$ State Key Laboratory of Proteomics, Beijing Proteome Research Center, Beijing Institute of Radiation Medicine, Collaborative \\ Innovation Center for Cancer Medicine, Beijing 100850, China \\ ${ }^{2}$ Department of Basic Medical Sciences, School of Medicine, Shihezi University, Shihezi, Xinjiang Province 832000, China \\ ${ }^{3}$ Department of Neuroscience and Regenerative Medicine, Medical College of Georgia, Augusta University, Augusta, GA \\ 30912, USA \\ Correspondence to: Lingqiang Zhang, email: zhangla@nic.bmi.ac.cn \\ Keywords: Smurfl, HECT domain, Ub binding region, ubiquitination, osteoblastic activity \\ Received: March 30, $2016 \quad$ Accepted: May 29, $2016 \quad$ Published: July 18, 2016 \\ Copyright: Zhang et al. This is an open-access article distributed under the terms of the Creative Commons Attribution License 3.0 \\ (CC BY 3.0), which permits unrestricted use, distribution, and reproduction in any medium, provided the original author and source \\ are credited.
}

\section{ABSTRACT}

The HECT-type ubiquitin ligase Smurf1 (Smad ubiquitination regulatory factor-1) plays the prominent role in regulation of bone formation, embryonic development, and tumorigenesis by directing the ubiquitin-proteasomal degradation of specific targets. In contrast with RING-type E3s, the catalytic HECT domain of Smurf1 firstly binds to and then transfers ubiquitin (Ub) molecules onto the substrates. The Smurf1Ub interaction is required for Smurf1 catalytic ligase activity to promote substrate degradation. However, so far specific regulators or compounds controlling Smurf1-Ub interaction and the ligase activity have not been identified. Here we report two small molecule compounds targeting Ub binding region of HECT domain interrupt Smurf1-Ub contact, inhibit Smurf1 ligase activity and stabilize BMP signal components Smad1/5 protein level. Furthermore, these compounds increase BMP signal responsiveness and enhance osteoblastic activity in cultured cells. These findings provide a novel strategy through targeting Smurf1 ligase activity to potentially treat bone disorders such as osteoporosis.

\section{INTRODUCTION}

Ubiquitination, which involves in the intracellular protein turnover of various biological processes, is essential for maintaining physiological function of organism and cellular homeostasis. The dysfunction of this process will lead to the disorders and disease of body, including cardiac dysfunction [1], bone degenerative disease [2], metabolic disorders [3], and cancer malignancy $[4,5]$. During ubiquitination, E3 ubiquitin ligases function as the final link to recognize and select substrate for protein degradation [6]. More than 600 different ubiquitin-protein ligases have been identified [6], which are classified into two types: the scaffold-type and the thioester bond intermediate-type [7-9]. The vast majority of E3 ligases belong to the former type, which contains a RING (Really Interesting New Gene) finger domain, whereas the latter form the E6AP carboxyl terminus (HECT) domain family [8]. RING E3 ligases ubiquitinate and degrade targets in an E2-dependent manner, during which process, the E2s and target proteins interact with E3s respectively. By the medium of E3s, E2s transfer ubiquitin (Ub) directly to specific internal Lys residues of those proteins. That means Ubs are delivered from E2 to target proteins and tagged onto the specific lysine residue of substrates. Unlike RING E3s, the HECT E3s interact with E2s and are responsible for the delivery of Ub. HECT E3s receive Ub molecules by the conserved HECT domain in the $\mathrm{C}$ terminus and form the Ub-thioester to transfer $\mathrm{Ub}$ onto subtracts for ubiquitination [10].

Smurf1, a C2-WW-HECT ubiquitin ligase, which belongs to the Nedd4 (neural precursor cell expressed developmentally downregulated gene 4) family is a key negative regulator of transforming growth factor (TGF)- $\beta /$ bone morphogenetic protein (BMP) signaling pathway [11-14] and also involves in regulation of other 
important biological pathways, such as the non-canonical Wnt pathway and the mitogen-activated protein kinase pathway. Therefore, multiple functions of Smurfl has been demonstrated in bone homeostasis [11, 12, 14], embryogenesis [15] and autophagy [16]. Smurf1 uses the WW domains to capture Smads, characterized the PY motif, and controls TGF- $\beta$ /BMP signaling transduction by degrading the components including Smad1, Smad4, Smad5 and Smad7 in the ubiquitination process [11, 14, 17, 18]. Meanwhile, to regulate cell growth and differentiation, Smurfl also has other substrates in the TGF- $\beta$ and BMP pathways, including RunX2, RunX3, Tbx6, MEKK2, JunB, and TRAF4 [19-23].

The maintenance of bone homeostasis depends on the dynamic regulation of two processes: bone formation and resorption, in which the osteoblast acts on promoting bone formation and bone increase. The differentiation of mesenchymal stem cells (MSCs) into osteoblasts and osteogenesis relies on the BMP signaling. As the components and related transcription factors of the BMP pathway Smads, MEKK2, RunX2 and JunB are crucial in regulation the differentiation and growth of osteoblasts. Therefore, as the negative factor of BMP pathway, Smurf1 is closely related to the osteoblast differentiation and postnatal bone formation. Smurfl $1^{-/-}$mice display bone mass increase in an age-dependent manner $[12,22]$.

In consideration of E3 activity and the physiological functions of Smurf1, targeting this negative regulator of BMP pathway will specifically regulate E3 activity, control signal transmission and affect pathological manifestations, which become a candidate strategy in future bone metastasis disease therapies. Up to now, a series of studies have shown that certain activators could enhance the E3 activity of Smurfland augment Smurfl-mediated ubiquitination, such as casein kinase 2 interacting protein-1 (CKIP-1) [22, 24] and Cdh1, which is identified as the activator of anaphase-promoting complex(APC) [10]. Furthermore, these activators reduce cellular responses to TGF- $\beta$ /BMP signaling, and depress the bone formation process and attenuate osteogenetic activity. Otherwise, a cullin E3 ligase complex named $\mathrm{SCF}^{\mathrm{FBXL} 15}$ could ubiquitinate Smurf1 and induce its proteasomal degradation [25]. As SCF $\mathrm{SBXL15}^{\mathrm{FB}}$ downregulates the protein level of Smurf1, it leads to the decline of E3 activity and increase of osteogenesis.

The critical role of Smurfl in the bone formation regulation has been extensively investigated and the relevant substratesin the BMP pathway also has been discovered. However, how to hold back Smurf1 receiving $\mathrm{Ub}$ and then regulate BMP pathway and osteoblastic activity is little known. In this study, we simulated the $\mathrm{Ub}$ binding region of Smurfl and identified two small molecule compounds via computer virtual screening. They specifically target the HECT domain of Smurf1 and interrupt Ub-Smurfl contact, thus inhibit the degradation of Smad1/5. Notably, this work further demonstrate these two compounds strengthen the bone synthesis ability by promoting BMP signal transduction. So far as we know, this is the first time to identify small molecule compound which specifically targets the HECT domain of Smurf1 ligase.

\section{RESULTS}

\section{Computer virtual screen compounds aim at HECT domain of Smurf1}

The structure of Smurf1 WW domains (WW1 and WW2) and a phospholipid binding C2 domain have been identified and analyzed, however the three-dimensional structure of Smurf1 catalytic HECT domain remains unclear. Given that both Smurf1 and Smurf2 belong to Nedd4 family, and their amino acid sequence homology of HECT domain is more than $90 \%$, the structure of this domain was obtained by protein modeling performed on PyMOL and referred to the counterpart domain of Smurf2 (Figure 1A). Based on the structure, we defined a concave region on the HECT domain, surrounded by Asn431, Tyr439, Asn481 and Gln653, as a hydrophobic pocket which is likely to key area for its combination with ubiquitin (Figure 1B). By computer high-throughput virtual screening, we acquired about 100 compounds that might target HECT domain of Smurf1 from more than one million available small molecule compounds (Figure 1C).

\section{Candidate compounds B06 and B75 enhance osteoblast differentiation via activating BMP signaling}

By high-throughput virtual screening and compounds skeleton classification, 24 representative compounds were selected in the top 100 scoring ones for the next preliminary selection (Supplementary Tables S1 and S2). Since Smurf1 could reduce cellular responses to bone morphogenetic protein (BMP) via triggering proteasomal destruction of Smad1 and Smad5, we made alkaline phosphatase (ALP) activity as chief standard in the preliminary selection, since it is one of the most canonical marker that can reflect bone formation ability in bone anabolism. For the subsequent assays, we selected mouse myoblasts cell line $\mathrm{C} 2 \mathrm{C} 12$, which possesses characteristic of mesenchymal stem cell and respond to BMP signal. We measured ALP activity after treatment with the compound $(2 \mu \mathrm{M})$ and BMP-2 $(50 \mu \mathrm{M})$ in the $\mathrm{C} 2 \mathrm{C} 12$ cells. A previously identified Smurf1 WW domain-targeting compound named A17 was used as a positive control in these assays [26]. The results showed that B06, B07, B10, B11 and B75 improved ALP activity compared with negative control DMSO (Figure 2A). These candidate compounds were further screened with analysis of Smad1/5 protein levels. After treatment with each of the examined compounds $(10 \mu \mathrm{M})$ and BMP-2 $(50 \mu \mathrm{M})$, the compounds B06 and B75 obviously up-regulated the protein level of Smad1/5 (Figure 2B). In addition, the mRNA levels of Smad1 and Smad5 were not significantly changed under the same treatment conditions with these 
A

B
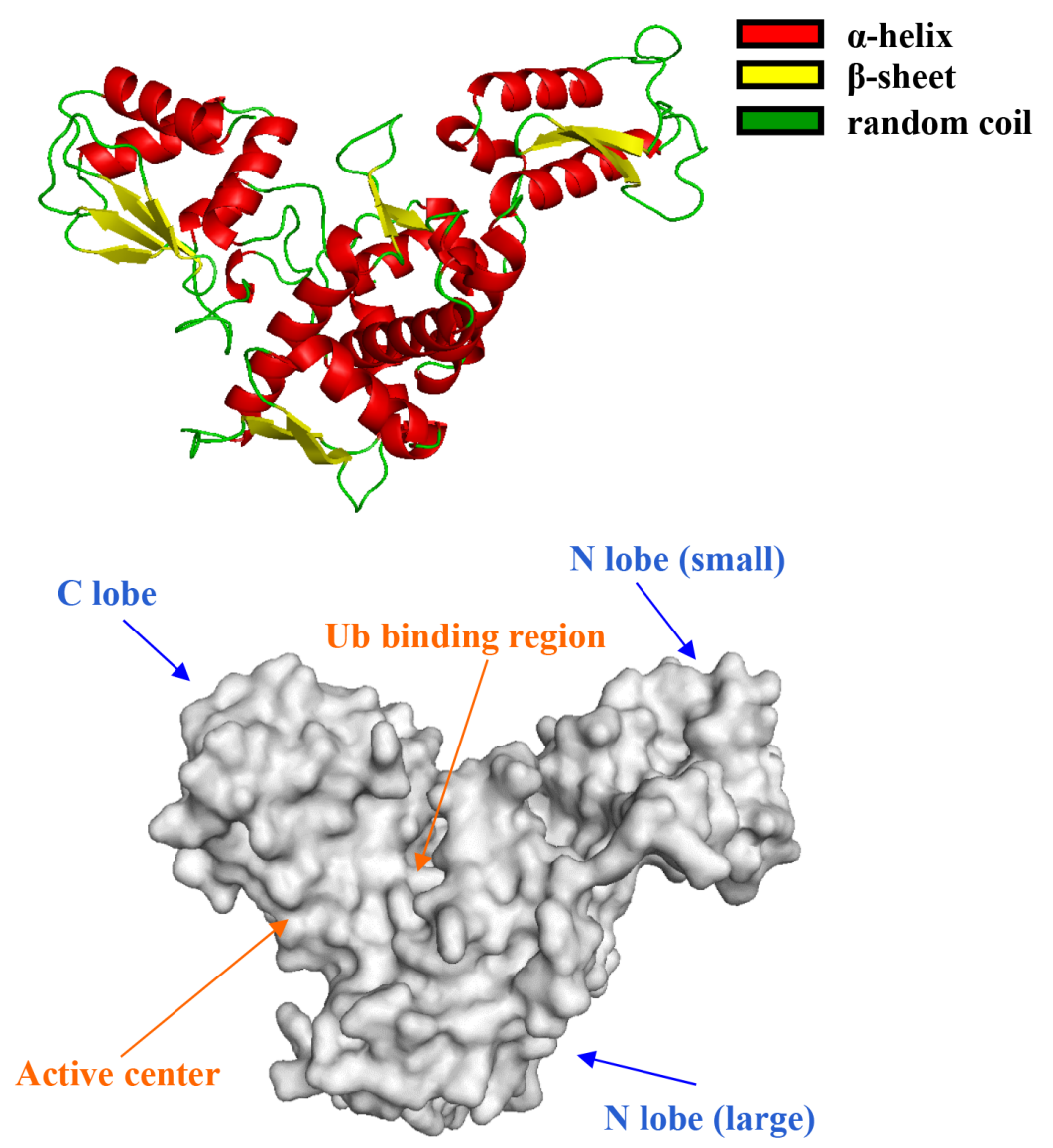

C

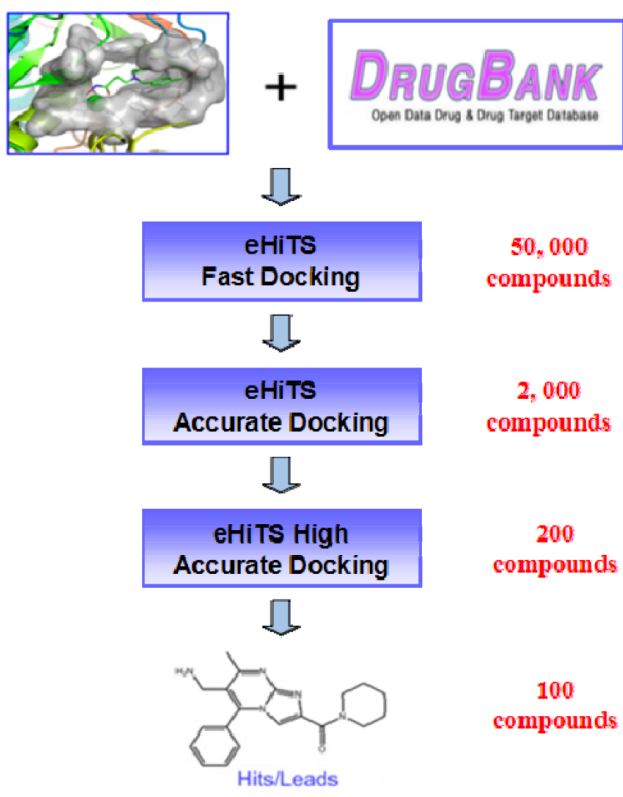

Figure 1: Computer virtual screen compounds targeting the HECT domain of Smurf1. A. The protein modeling of Smurf1 HECT domain. The $\alpha$-helix (red), $\beta$-sheet (yellow) and random coil (green) were labeled in HECT domain. B. The surface structure of Smurf1 HECT domain. The Ub binding region (orange arrow), active center (orange arrow) and two lobe (blue arrows) were point out by different arrows. C. Work-flow of computer virtual screening. 


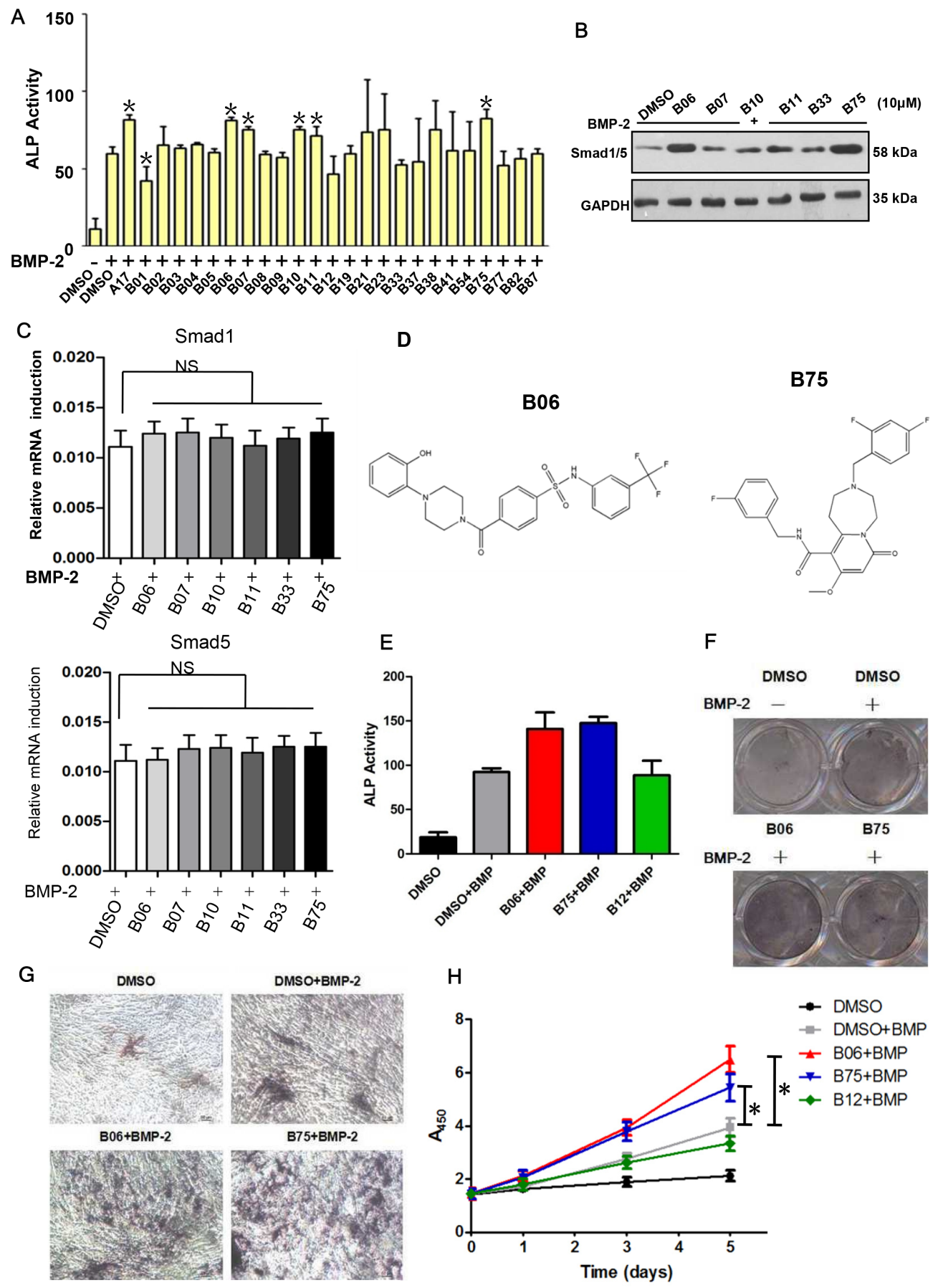

Figure 2: Candidate compounds B06 and B75 promotes the bone synthesis ability via activating BMP signaling. A. The effection of candidate compounds to ALP activity. C2C12 cells were deal with DMSO $(0.1 \%)$ or compound $(2 \mu \mathrm{M})$, then it were stimulated by BMP-2 $(50 \mathrm{ng} / \mathrm{ml})$ after $1 \mathrm{~h}$, ALP activity test wound be executed after $48 \mathrm{~h}$. B. The effection of candidate compounds to the expression of Smad1/5 protein. C2C12 cells were deal with DMSO $(0.1 \%)$ or compound $(10 \mu \mathrm{M})$, then it were stimulated by BMP-2 (50ng/ $\mathrm{ml}$ ) after $1 \mathrm{~h}$, the proteins expression wound be detected after $8 \mathrm{~h}$ by western blot. All of the above data points were determined in triplicate and showed with the mean $\pm \mathrm{SD}(*: p \leq 0.05$, t-test). C. $\mathrm{C} 2 \mathrm{C} 12$ cells were treated B06 and B75 at $2 \mathrm{mM}$, while BMP-2 was used at $50 \mathrm{ng} /$ $\mathrm{ml}$. Data points were determined in triplicate and showed with the mean $\pm \mathrm{SD}\left({ }^{*}: \mathrm{p} \leq 0.05\right.$, t-test). D. Chemical structures of B06 and B75. E. Selective compounds enhance BMP-2 induced ALP activity. C2C12 cells were dealed with DMSO $(0.1 \%)$ or compound $(2 \mu \mathrm{M})$, then it were stimulated by BMP-2 (the concentration is $100 \mathrm{ng} / \mathrm{ml}$ ) after $1 \mathrm{~h}$. ALP activity test was performed after $48 \mathrm{~h}$. Data points were determined in triplicate and showed with the mean $\pm \mathrm{SD}(*: p \leq 0.05$, t-test). F. Selective compounds enhance BMP-2 induced ALP content. C2C12 cells were dealed with DMSO $(0.1 \%)$ or compound $(2 \mu \mathrm{M})$, then it were stimulated by BMP-2 $(100 \mathrm{ng} / \mathrm{ml})$ after $1 \mathrm{~h}$, ALP colouration test was performed after 48h. G. ALP staining results were shown under microscope. H. Selective compounds can obvious promote BMP-2 induced cells proliferation. Data points were determined in triplicate and showed with the mean $\pm \mathrm{SD}(*: p \leq 0.05$, t-test). 
compounds, suggesting that B06 and B75 specifically affect Smad1/5 protein level (Figure 2C). Meanwhile, we found that both B06 and B75 fit the rules of Lipinski and possess distinct properties (such as structure) (Figure 2D and Supplementary Table S3). Based on the above results, we speculated that the small molecule compounds B06 and B75 might be the best candidates among the examined compounds. To verify the effects of B06 and B75 in promoting bone synthesis in $\mathrm{C} 2 \mathrm{C} 12$ cells, we performed ALP activity assays again under BMP-2 stimulation with a high-does treatment $(50 \mu \mathrm{M})$. Compared with the control group, both B06 and B75 enhanced ALP activity, whereas another compound B12 did not (Figure 2E). Furthermore, we performed ALP staining assays, and confirmed that they were able to increase the intracellular ALP content under BMP-2 stimulation (Figure $2 \mathrm{~F}$ and $2 \mathrm{G}$ ). In order to investigate the effect of $\mathrm{B} 06$ and $\mathrm{B} 75$ on $\mathrm{C} 2 \mathrm{C} 12$ cell proliferation, we performed WST-1 cell proliferation and cell toxicity test. The results showed that both B06 and B75 can promote $\mathrm{C} 2 \mathrm{C} 12$ cells proliferation, and their toxicity was low (Figure 2H). Taken together, these data suggest B06 and B75 can enhance the osteoblast differentiation of C2C12 cells.

\section{B06 and B75 inhibit Smurf1-mediated Smad1/5 ubiquitination and degradation}

We confirmed that the selective compounds B06 and B75 upregulated the Smad1/5 protein levels (Figure $3 \mathrm{~A}$ ) and both compounds increased Smad1/5 protein level in a dose-dependent manner (Figure 3B). Since S206 of Smad1 was phosphorylated modified in BMP-2 signaling pathway, we tested the expression of p-Smad1 (S206) and confirmed that B06 and B75 are able to upregulate its level (Figure 3C). We also performed protein decay experiment and the subsequent test revealed that B06 and B75 could prolong the half-life of endogenous Smad1/5 under BMP2 stimulation (Figure 3D). Next, we compared the effect of selective compounds and proteasome inhibitor MG132. Under BMP-2 stimulation, B06 and B75 were able to upregulateSmad1/5 protein level which effect was similar to that of MG132 (Figure 3E). Furthermore, we performed a Smad1/5 ubiquitination assay in vivo to test whether B06 and B75 stabilize Smad1/5 protein level via inhibiting Smurf1-mediated Smad1/5 ubiquitination. The result showed that B06 and B75 strongly inhibited Smad1/5 ubiquitination under rhBMP-2 stimulation compared with control (Figure 3F).

\section{B06 and B75 interrupt interaction between Smurf1 and Ub but not Smurf1 and Smad1/5}

Given the screen rationale, we next investigated whether B06 and B75 weaken or block the direct interaction between Smurfl and Ub, the binding assay in vitro was performed. The pull-down results showed that single Ub protein can be readily copurified with GST-
Smurf1, and incubation with B06 and B75 interrupted Smurfl and Ub binding. Since the amino acid sequence homology of Smurf1 and Smurf2 HECT domains are more than $90 \%$ and Smurf2 also contains a Ub-binding region to capture Ub molecules, we tested the effect of B06 and B75 on interaction between Smurf2 and Ub. However, B06 and B75 did not interrupt the interaction between Smurf2 and $\mathrm{Ub}$ (Figure 4A). We further examined the possible impacts of the two compounds on Smurf1-Smad and Smurf1-E2 interaction. The Smurf1-Smad1 interaction assay was performed that exogenous Smad1 was transfected into HEK293T cells with Smurf1-CA mutant, which abolishes ubiquitin ligase activity and fails in ubiquitination by changing the HECT domain crucial site Cys699 to an Ala. However, this point mutant still reserves binding ability to its interacting proteins. Co-immunoprecipitation of Smad1 showed that both selective compounds B06 and B75 had no effect on Smurfl interaction with Smad1 (Figure 4B). Similarly, an in vitro binding assay was performed between Smurf1 and its E2s, UbcH5c and UbcH7, which interact with the HECT domain of Smurfl and deliver the ubiquitins onto it. The result showed that selected compounds did not interrupt Smuf1-E2 interaction (Figure 4C). In conclusion, B06 and B75 specifically interfere with the interaction between Smurfl and $\mathrm{Ub}$ but not Smurf1 and Smad1/5. We also tested the possible effect of the compounds on Smurf2 with the substrates $\mathrm{Smad} 2 / 3$. The results showed that Smurf 2 downregulated the protein level of Smad2/3, as expected, however, B06 and B75 had no inhibitory effects on the degradation (Figure 4D). Subsequently, the effect of both compounds on the interactions of Smurf2-Smad2/3 were tested via in vivo co-immunoprecipitation assays. The results showed that B06 and B75 could not interrupt Smurf2-Smad2 or Smurf2-Smad3 interactions (Figure 4E), indicating that both compounds might act specifically on Smurf1.

\section{B06 and B75 control Smad1/5 and other targets in a Smurf1-dependent manner}

In vivo assays revealed that B06 and B75 could elevate Smad1/5 when cells were pre-transfected wild type Smurf1 but not the C699A (Smurf1 CA) mutant (Figure $5 \mathrm{~A})$. To identify whether the compounds affect Smad1/5 in a Smurf1-dependent manner, we knocked down Smurf1 by specific siRNA under rhBMP-2 stimulation. We found that neither B06 or B75 could elevate Smad1/5 protein level (Figure 5B), indicating the dependence of Smurf1. The fact that B06 and B75 interrupt the interaction between Smurf1 and Ub implies that they might inhibit Smurf1mediateddegradation of other substrates. To verify this point, we examined other reported substrates of Smurf1 besides Smad1/5. It turned out that both B06 and B75 upregulated the protein level of Runx2, Smad2/3, Smad4 and ING2 (Figure 5C), although their binding modes with Smurf1 are incompletely identical. That confirmed that B06 and B75 specifically inhibit Smurf1-mediated 
A

A

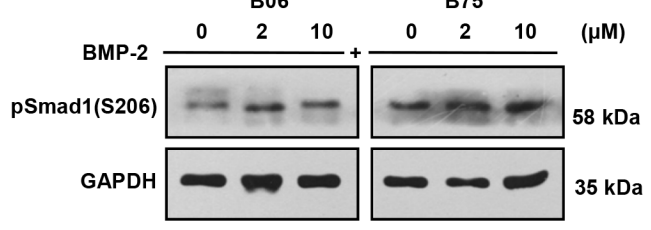

D
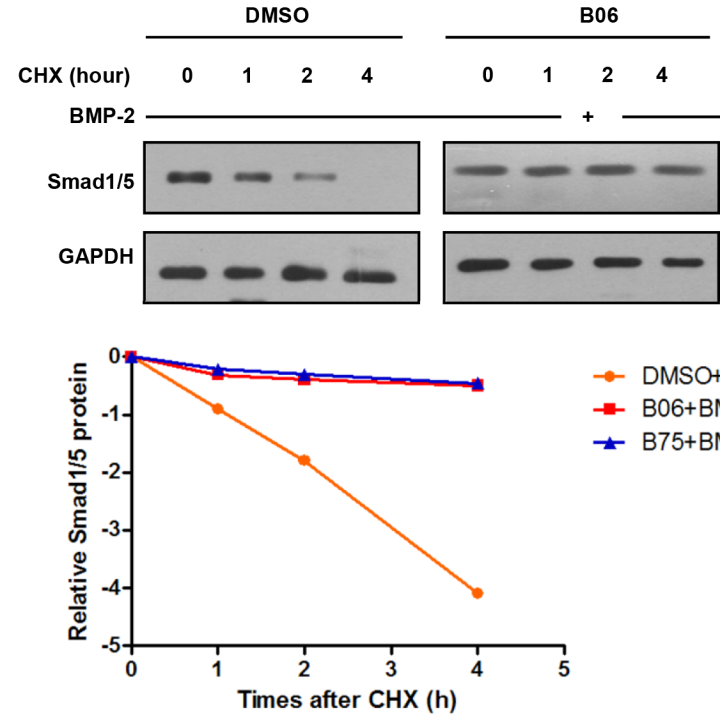

$\mathrm{E}$

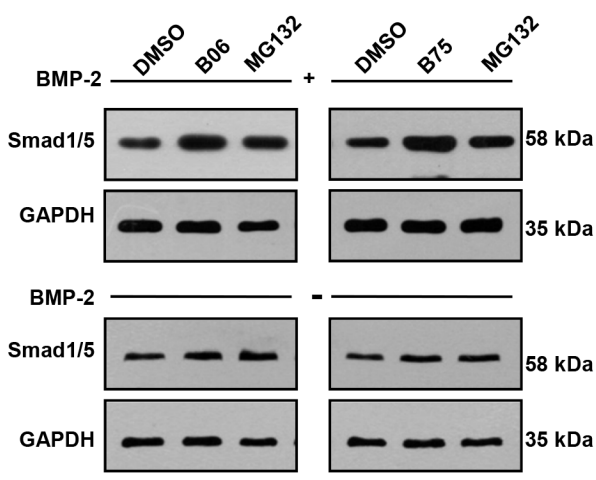

$-\mathrm{B} 06+\mathrm{BMP}$

- B75+BMP
B

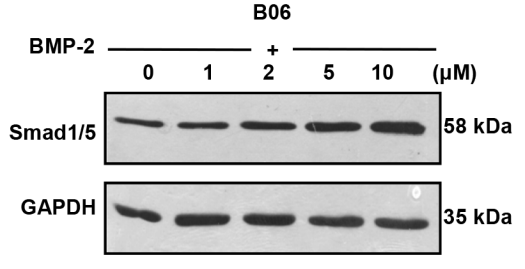

B75
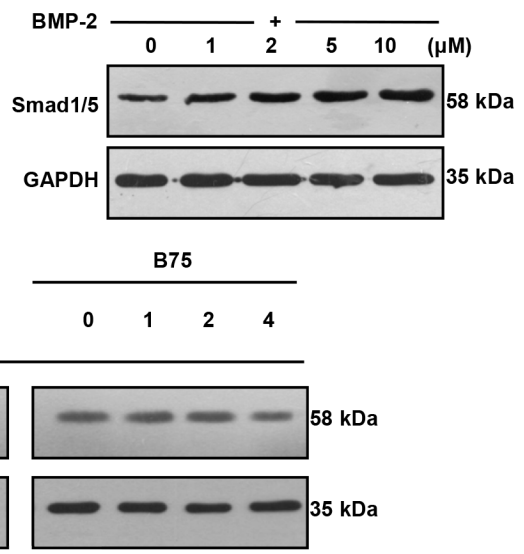

$\rightarrow$ DMSO+BMP
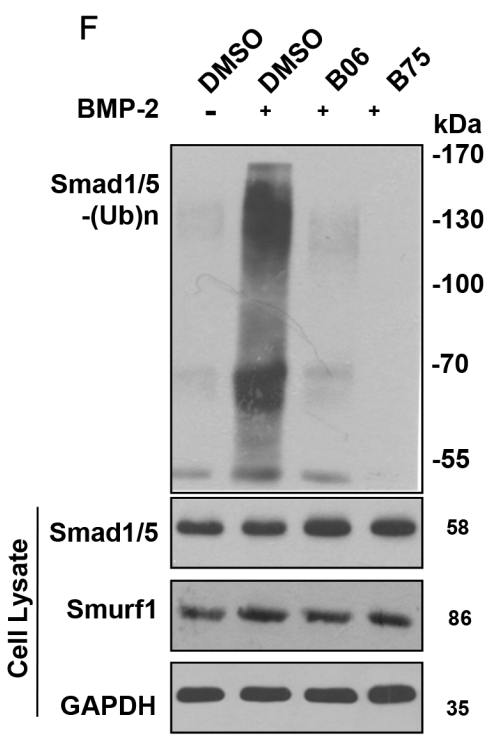

Figure 3: B06 and B75 inhibit Smurf1-mediated Smad1/5 ubiquitination and degradation. A. C2C12 cells were treated with B06 and B75 $(2 \mu \mathrm{M})$ together with rhBMP-2 $(50 \mathrm{ng} / \mathrm{ml})$ or not. GAPDH were used as loading controls. B. Selective compounds can increase the Smad $1 / 5$ protein level. C2C12 cells were dealed with DMSO $(0.1 \%)$ or compound (the concentration is $1 \mu \mathrm{M}, 2 \mu \mathrm{M}, 5 \mu \mathrm{M}$ and $10 \mu \mathrm{M})$, then it were stimulated by BMP-2 $(50 \mathrm{ng} / \mathrm{ml})$ after $1 \mathrm{~h}$, the proteins expression was detected after $8 \mathrm{~h}$ by WB. C. Selective compounds can increase Smad1 phosphorylation (S206) level. C2C12 cells were dealed with compound ( $2 \mu \mathrm{M}$ ), and stimulated by BMP-2 $(50 \mathrm{ng} / \mathrm{ml})$; after $1 \mathrm{~h}$, the p-Smad1(S206) protein level was detected after $8 \mathrm{~h}$ by WB. D. Selective compounds prolonged the half-time of Smad $1 / 5$ protein. C2C12 cells were dealed with DMSO $(0.1 \%)$ or compound $(2 \mu \mathrm{M})$, then stimulated by BMP-2 $(50 \mathrm{ng} / \mathrm{ml})$ and CHX (10 $\mu \mathrm{g} / \mathrm{ml})$; after $1 \mathrm{~h}$, the Smad $1 / 5$ protein expression was detected by WB after the indicated times $(0,1,2$ and $4 \mathrm{~h})$. The data were analyzed through software Image J and GraphPad Prism. E. Detection of Smad1/5 protein level following selective compounds or proteasome inhibitor (MG132) treatments. F. Selective compounds impeded the ubiquitination of Smad1/5. C2C12 cells were treated B06 and B75 at $2 \mu \mathrm{M}$, while MG132 and rhBMP-2 were used at $20 \mathrm{mM}$ and $50 \mathrm{ng} / \mathrm{ml}$. GAPDH were used as loading controls. 


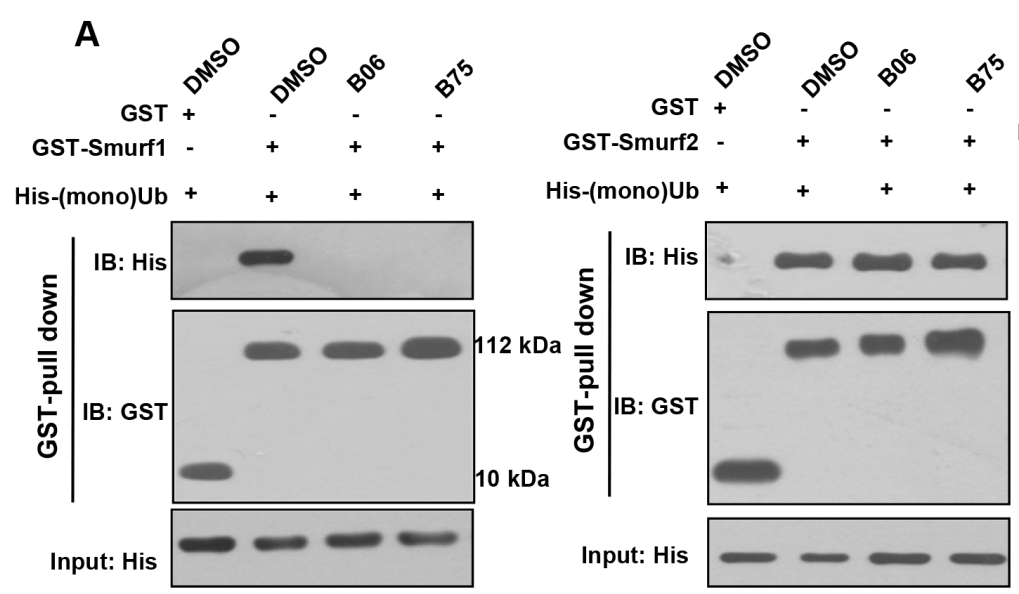

B

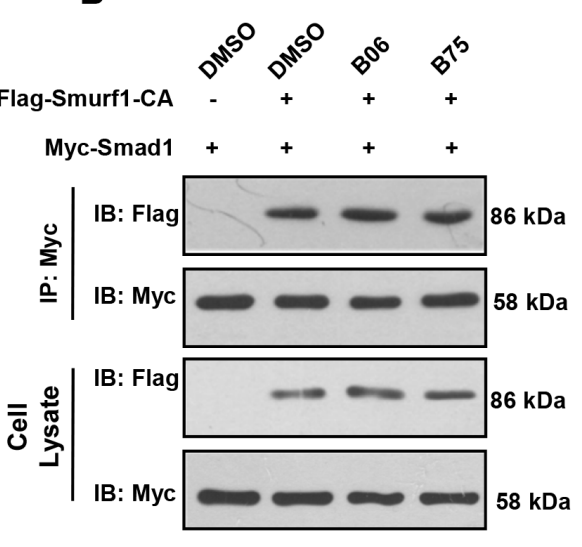

C

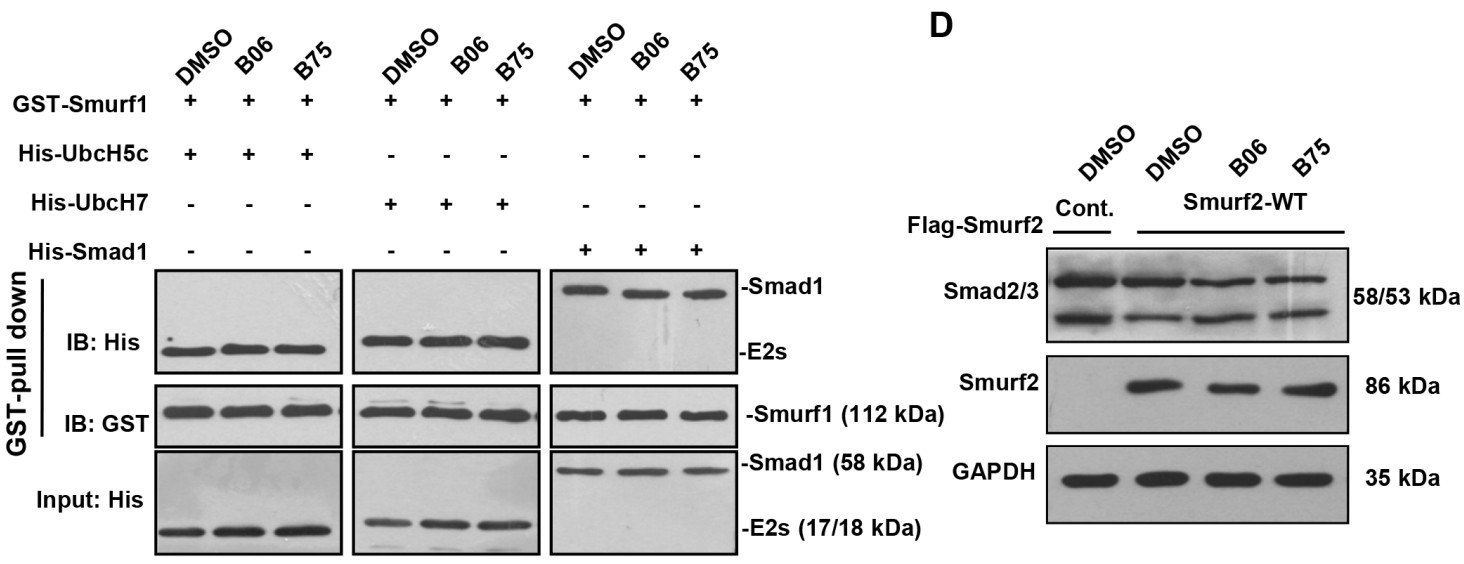

$\mathbf{E}$

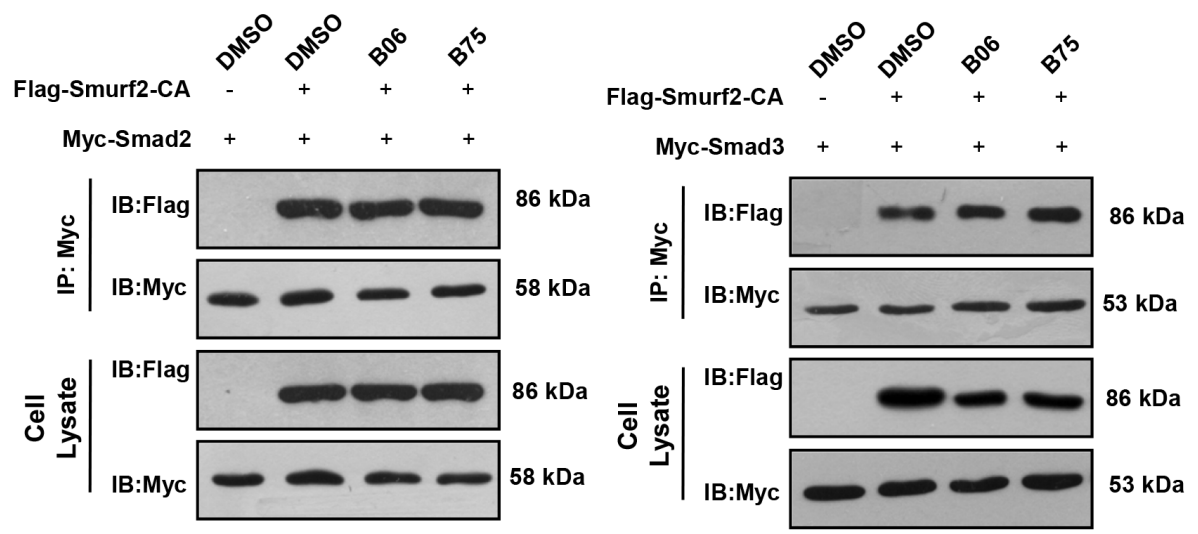

Figure 4: B06 and B75 compounds interrupt the interaction between Smurf1 and Ub. A. GST pull-down assays were performed to show that GST-tagged Smurf1 and Smurf2 directly interacts with mono-Ub in vitro, and B06 and B75 specifically inhibit Smurf1-Ub binding. B. Selective compounds had no significant effects on Smurf1-Smad1 interaction. 293T cells were co-transfected with Flag-empty vector and Myc-Smad1 (lane 1) or Flag-Smurf1-CA and Myc-Smad1 (lanes 2-4) plasmids. For inhibitors administration, cells were treated with B06 and B75 at $2 \mu \mathrm{M}$. C. Selected compounds do not interrupt Smuf1-E2 contact. Prokaryotic expressed proteins were purified and employed in GST-pull down. B06 and B75 were used at 10mM. Note that cropped blots are shown here. D. 293T cells were transfected Flag-empty vector (lane 1) and Flag-Smurf2 (lane 2). For inhibitors administration, cells were treated with B06 and B75 at $2 \mu \mathrm{M}$ (lanes 3-4). E. Left: 293T cells were co-transfected with Flag-empty vector and Myc-Smad2 (lane 1) or Flag-Smurf2-CA and Myc-Smad2 (lanes 2-4) plasmids. Right: 293T cells were co-transfected with Flag-empty vector and Myc-Smad3 (lane 1) or Flag-Smurf2-CA and MycSmad3 (lanes 2-4) plasmids. For inhibitors administration, cells were treated B06 and B75 at $2 \mu \mathrm{M}$. Note that cropped blots are shown here. 
A
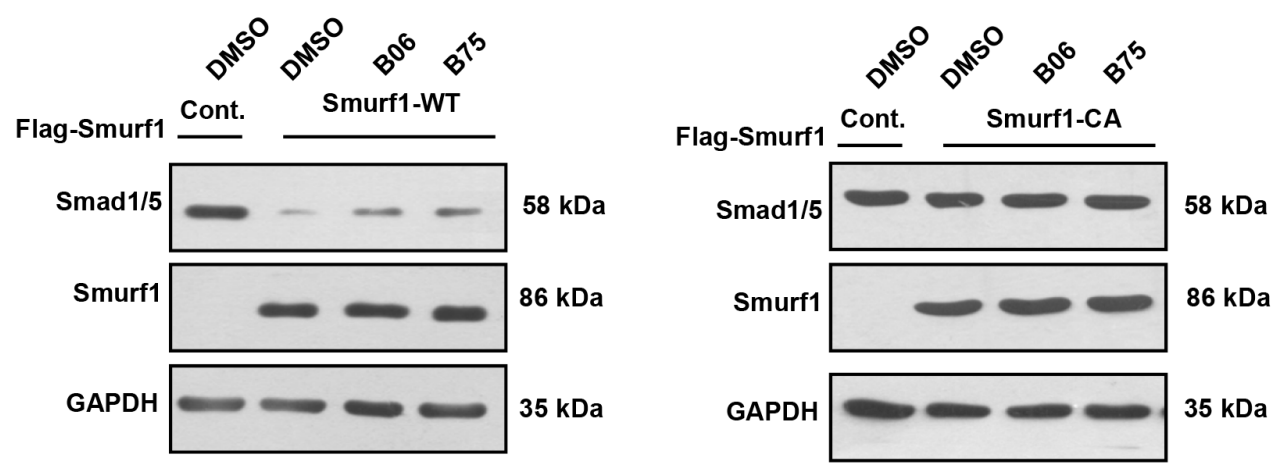

B

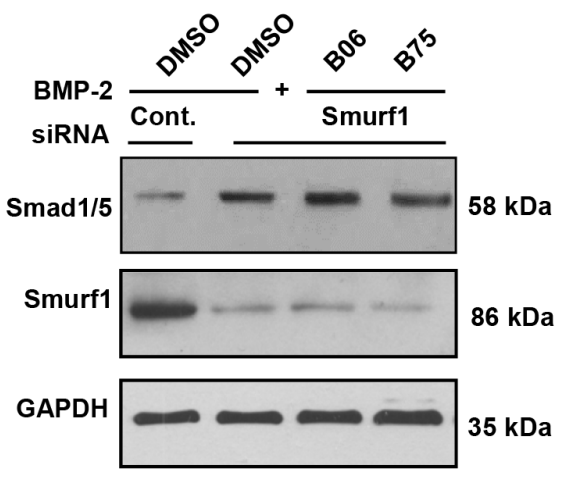

C

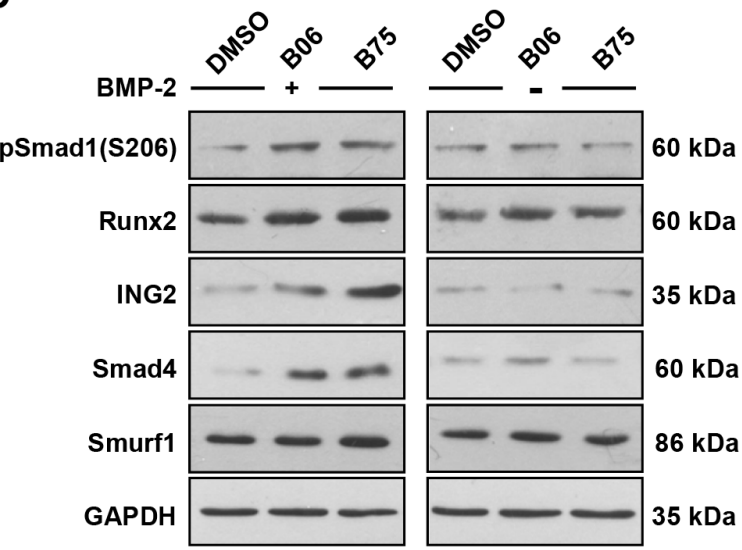

D
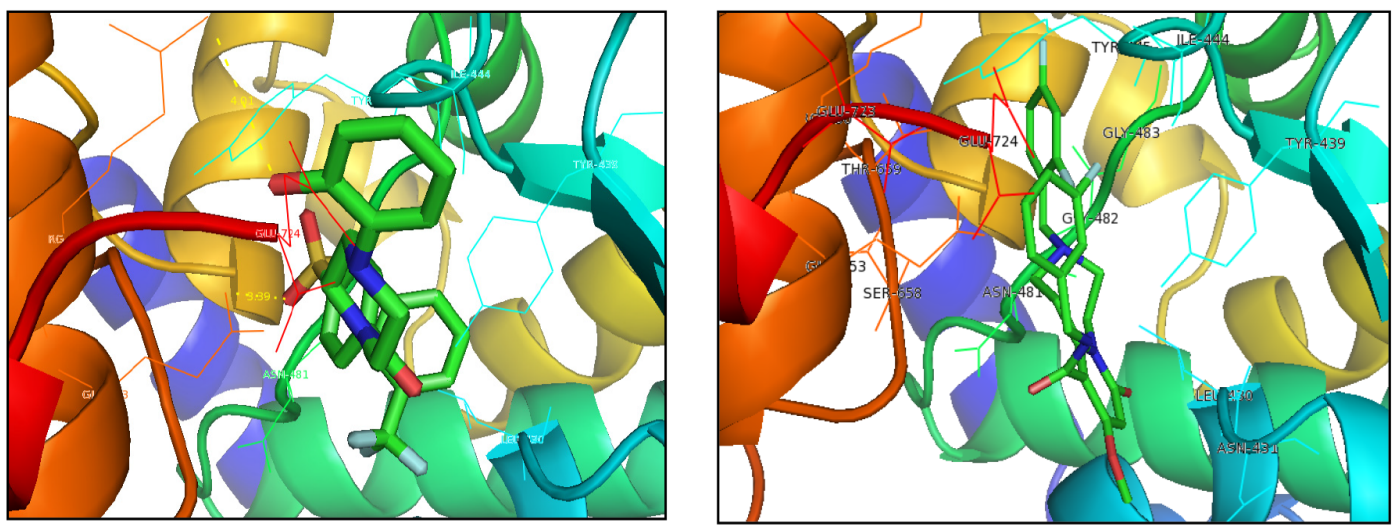

\section{Predicted binding modes of B06 with Ub binding region}

\section{Predicted binding modes of B75 with Ub binding region}

Figure 5: B06 and B75 control Smad1/5 and other targets in a Smurf1-dependent manner. A. 293T cells were transfected with the indicated plasmids. For inhibitors administration, cells were treated with B06 and B75 at $2 \mu \mathrm{M}$. B. Smurfl was knocked down in $\mathrm{C} 2 \mathrm{C} 12$ cells (lane 1: control siRNA, lanes 2-4: mouse Smurf1 siRNA). For inhibitors administration, cells were treated with B06 and B75 at $2 \mu \mathrm{M}$, while rhBMP-2 was used at $50 \mathrm{ng} / \mathrm{ml}$. GAPDH were used as loading controls. Note that cropped blots are shown here. C. Effects of selective compounds on other substrates of Smurf1. D. Predicted binding mode of B06 with the defined Ub binding region. Key residues in the region were labeled in different colours. The distances (angstrom) of hydrogen bonds donors and receptors were noted in numerical values and yellow dotted lines. Predicted binding modes of B75 with the defined Ub binding region. Key residues in the region were labeled in black. 
ubiquitin-proteasomal degradation rather than impairs the substrate binding. Finally, we predicted the binding modes of B06 and B75 with the defined $\mathrm{Ub}$ binding region for intensive research (Figure 5D).

\section{DISCUSSION}

In this study, we screened small molecules which specifically targeted the ubiquitin-binding site within Smurf1 HECT domain. A typical HECT domain consists of two lobes: N-lobe interacts with the E2 and C-lobe contains the active-site cysteine that forms the thioester bond with ubiquitin [27]. Interestingly, Smurf1 and Smurf2 also contain a non-covalent ubiquitin binding region within the $\mathrm{N}$-lobe, which is required for their E3 ligase activity. We established the strategy that screened compounds through computer virtual and found the best match compounds B06 and B75 inhibiting the combination between Smurfl and $\mathrm{Ub}$ could enhance BMP signaling and promote $\mathrm{C} 2 \mathrm{C} 12$ cells differentiation and proliferation. This strategy specifically targeted the $\mathrm{Ub}$ binding region of HECT domain, and did not affect the interaction of Smurf1 and E2. Therefore, it is a novel strategy to inhibit the ligase activity and control the Smurf1-mediated degradation.

As mentioned above, Smurf1 controls the turnover of components of BMP signaling cascades, which is a negative regulator of osteoblast differentiation and bone formation. The identified mechanisms regulating Smurf1 include the transcriptional and post-transcriptional level. At the transcriptional level, tumor necrosis factor (TNF) increases Smurf1 expression by elevating Smurf1 transcription and inhibits osteoblastic bone formation [28-30]. At post-transcriptional level, nuclear protein kinase CK1 $\alpha$ stabilizes the expression of Smurf1 by regulating the expression of vertebrate-specific pre-mRNA binding protein heterogeneous nuclear ribonucleoprotein (hnRNP-C) [31]. In mesenchymal stem cells, microRNA-17 directly targeting Smurf1 3'UTR hinder the E3 ligase expression and enhance osteogenesis [30]. Moreover, we have previously shown that Smurf1 expression and activity are also regulated by CKIP-1, an activator augments the E3 ligase activity by targeting its linker region between the two WW domains, thereby promoting Smurf1-mediated ubiquitination [24]. We subsequently established a new approach targeting CKIP-1 to treat metabolic skeletal disorders, which uses (AspSerSer)6-liposome to deliver siRNAs specifically to bone-formation surfaces. This target delivery system selectively stimulates bone formation without affecting bone resorption [32]. Besides CKIP-1, others find that Cdh1, another activator interacting with the C2 and WW1 domains of Smurf1, disrupts the auto-inhibitory Smurf1 dimers and promotes the E3 ligase activity of Smurf1 (10). On the other hand, a series of E3 ligases including $\mathrm{SCF}^{\mathrm{FBXL} 15}$, and Smurf2 were shown to trigger ubiquitin- proteasomal degradation of Smurf1 [25, 33]. Recently, we analyzed the reported co-structure of Smad1 and Smurf1 WW domains interaction, which affects Smurfl activating Smad1, and identified a binding hydrophobic pocket providing the position for this interaction. Ultimately, we discovered the small molecule compounds A01 and A17 blocking the WW1 domain interacting with Smad1 based virtual screening [26]. We draw inspiration from this successful strategy and design the similar and distinctive scheme for screening the small molecule compounds targeting $\mathrm{Ub}$ binding region of Smurf1 instead of the interaction between Smurf1 and substrates.

A previous study showed that both Smurfl and Smurf2 HECT domains exihibit ubiquitin-binding surfaces for promoting substrate poly-ubiquitination [34]. Mutations in Tyr459 and Gly404 weaken the E3 activity of Smurf2, which indicates these two sites in the hydrophobic surface of the $\mathrm{N}$ lobe might exist Ub-binding region to capture $\mathrm{Ub}$ molecules for Smurf2-mediated ubiquitination as well as Smurf1. So far nine E3 ligase have been identified belonging to the Nedd4 family: Nedd4, Nedd4L, WWP1, WWP2, NEDL1, NEDL2, Itch, Smurf1 and Smurf2. On one hand, they are jointly involved in regulation of a distensible substrate network. On the other hand the multiple functions of these ubiquitin ligase have been explored in different biological and physiological process include embryonic development, the immune response and tumor invasion. Nedd4 as the specific E3 ligases directs ubiquitination of the tumor suppressor PTEN and positively regulates cell growth and development [8, 35]. Nedd4L, reveals similar functions with Nedd4, such as in viral budding and endocytosis [36, 37]. In addition, Nedd4L also targets Smad2, Smad4 and the TGF- $\beta$ receptor for ubiquitination of these substrates [38]. Itch plays a vital role in Th2 cell differentiation [34]. WWP1 promotes ubiquitination of RunX2 and have been identified a negative regulators of bone formation [39]. Furthermore, other Nedd4 family members have the partial overlapping functions in regulating TGF- $\beta$ and BMP pathways besides Smurf1. Therefore, whether these members possess the Ub binding region or not? If they had, we would apply the novel strategy we established for searching the small molecule compounds which could disrupt the interaction between them and $\mathrm{Ub}$, and control the relevant biological processes.

The finding of the function of B06 and B75 on inhibiting Smurf1-Ub contact also raises certain questions which should be addressed in future studies. For example, we still need develop an experimental animal model to test and verify the effects of the compounds on bone mass in vivo. In addition, whether the compounds are toxic or have undesirable side effects on organism is also worthy of further investigation.

In conclusion, our findings reveal a novel mechanism and strategy to search the small molecule compounds which target $\mathrm{Ub}$ binding region of the HECT 
E3 ligase Smurf1 and block the interaction between HECT domain and $\mathrm{Ub}$. The study provides a new and original strategy to control the activity of HECT E3 ligase.

\section{MATERIALS AND METHODS}

\section{Cell culture and reagents}

mouse myoblast cell line $\mathrm{C} 2 \mathrm{C} 12$ cells and human embryonic kidney cells $293 \mathrm{~T}$ cells were cultured in DMEM supplemented with 5\% fetal bovine serum (FBS). Cells were transfected with TurboFect (R0531, Thermo Scientific). BMP-2 was purchased from PeproTech. Compounds were purchased from JK chemical. Proteasome inhibitor MG132 was obtained from Sigma. FBS was obtained from Hyclone. DMEM was purchased from Corning.

\section{Antibodies}

All antibodies were purchased as follows: Antibodies against Smurf1 (ab38866, Abcam), antiSmad1/5 (ab75273, Abcam), anti-Smad2/3 (\#8685, CST), anti-Smad4 (\#9515, CST), anti-pSmad1(Ser206) (\#13820, CST), anti-Runx2 (ab23981, Abcam), anti-ING2 (ab109504, Abcam), anti-Myc(MBL), anti-GST(MBL), anti-His (MBL), anti-HA (MBL), anti-GAPDH (MBL), anti-Flag (MBL) and mouse/rabbit IgG (Santa Cruz).

\section{Computer virtual screening}

The structures of Smurf1 HECT domain was obtained by protein modeling performed on PyMOL, referred to the counterpart domain of Smurf2. Afterwards, a large compounds pool was be comprised of form four chemistry industrial companies: InterBioScreen Ltd., ChemBridge Corporation, ENAMINE Ltd. and Life Chemicals Inc. Then, all compounds were evaluated the drug ability by ADMET Predictor (Simulations Plus Inc. USA), on which a cutoff line was set to eliminate the compounds with ADMET risk score of $\leq 2$, to shrink this pool to smaller virtual library of about one million compounds. The target pocket was defined by the docking program eHiTS (SimBioSys Inc. Canada), and the virtual ligand auto-docking was performed by three hierarchical steps: (1). Fast docking. The step abated the pool to 50,000 compounds. (2). Accurate docking. The step abated the pool to 2,000 compounds. (3). High accuracy docking. The step abated the pool to 200 compounds. The eHiTS score $(\log \mathrm{Kd})$ of each ligand was generated simultaneously after these procedures. The docking conformation of each ligand and the pocket was visualized by CheVi (SimBioSysInc Canada). Finally, we obtained the top 100 scoring ones.

\section{Western blot}

Whole cell lysates were prepared according to the manufacturer's instructions. Protein samples were subjected to SDS-PAGE, and electrophoresed proteins were subsequently transferred onto a PVDF membrane. Membranes were blocked with 5\% nonfat dry milk in Trisbuffered saline with $0.1 \%$ Tween 20 and incubated with the indicated primary antibody, which was followed by incubation with an HRP-conjugated secondary antibody. Immune complexes were visualized with the Supex reagent, and luminescence was detected. Before assays, cells were cultured with the administration of compounds and BMP-2. In addition, cells were harvested 8 hours after compounds administration.

\section{Co-immunoprecipitation assay}

Transfection was performed using TurboFect(R0531, Thermo Scientific) following the manufacturer's instructions. After $48 \mathrm{~h}$ harvested, cells were lysed in HEPES lysis buffer (20 mM HEPES, pH 7.2, $50 \mathrm{MmNaCl}, 0.5 \%$ Triton $\mathrm{X}-100,1 \mathrm{mMNaF}$ and 1 mM DTT) supplemented with protease inhibitor cocktail (Roche). Immunoprecipitations were performed using the indicated primary antibody for $3-4 \mathrm{~h}$ and protein $\mathrm{A} / \mathrm{G}-$ agarose beads (Santa Cruz) overnight at $4^{\circ} \mathrm{C}$. The resulting immunoprecipitates were washed at least three times in HEPES lysis buffer. Lysates and immunoprecipitates were examined using the indicated primary antibodies followed by detection with the related secondary antibody and the SuperSignalchemiluminescence kit (Thermo).

\section{Protein half-life assay}

For Smad1/5 half-life assay, when $\mathrm{C} 2 \mathrm{C} 12$ cells in $20 \mathrm{~mm}$ plates reached about $60 \%$ confluence. Cells were cultured with the administration of compounds and BMP2. Eight hours later, cells were treated with the protein synthesis inhibitor cycloheximide $(10 \mu \mathrm{g} / \mathrm{ml})$ for the indicated durations before harvest.

\section{In vivo ubiquitination assay}

C2C12 cells were cultured in $100 \mathrm{~mm}$ medium reached about $60 \%$ confluence with the administration of compounds and BMP-2. After $12 \mathrm{hr}$, cells were treated with $20 \mu \mathrm{M}$ proteasome inhibitor MG132 (Calbiochem) for $8 \mathrm{hr}$. The cells were washed with PBS, pelleted, and lysed in $0.4 \mathrm{ml}$ of HEPES buffer (20 mM HEPES, $\mathrm{pH}$ 7.2, $50 \mathrm{mMNaCl}, 1 \mathrm{mMNaF}, 0.5 \%$ Titon-X100) plus $0.1 \%$ SDS, $20 \mu \mathrm{M}$ MG132 and protease-inhibitor cocktail. The lysates were centrifuged to obtain cytosolic proteins. Briefly, individual samples were incubated with anti-Smad1/5 antibody (Abcam) for 3 hand protein 
A/G-agarose beads (Santa Cruz) for a further $8 \mathrm{~h}$ at $4^{\circ} \mathrm{C}$. Then the beads were washed thrice with HEPES buffer. The proteins were released from the beads by boiling in $40 \mathrm{ml}$ of $2 \times$ SDS-PAGE sample buffer for $10 \mathrm{~min}$. Ten microliters of the samples were subjected to immunoblot against anti-HA monoclonal antibody (MBL) in individual experiments.

\section{GST pull-down}

To detect the direct binding of Smurfl with Ub, bacteria-expressed GST, GST-Smurf1 proteins were immobilized on Glutathione-Sepharose 4B beads (Amersham Biosciences) and washed, and then beads were incubated with His-Ub for $8 \mathrm{~h}$ at $4^{\circ} \mathrm{C}$ under rotation. Beads were washed with GST-binding buffer $(100 \mathrm{mMNaCl}$, $50 \mathrm{mMNaF}, 2 \mathrm{mM}$ EDTA, 1\% NP-40 and protease inhibitor mixture) and proteins were eluted, followed by immunoblotting. The direct binding assay of Smurf1Smad1, Smurf1-UbcH5c, Smurf1-UbcH7, Smurf2-Ub are the same with this assay.

\section{ALP activity assay and ALP staining}

Cells were cultured in $100 \mathrm{~mm}$ medium for three days and administrated with compounds and rhBMP-2 on the day 3. ALP activity was examined by Alkaline Phosphatase, Diethanolamine Detection Kit (Sigma-Aldrich) following the manufacturer's protocols. ALP staining was performed by BCIP/NBT Alkaline Phosphatase Color Development Kit (Beyotime) following the manufacturer's protocols. Cells were stained on the 6th day.

\section{Cell counting assay}

Cell counting assay was performed by WST-1 Cell Proliferation and Cytotoxicity Assay Kit (Beyotime) following the manufacturer's protocols.

\section{Molecular visualization and statistical analysis}

Protein and compounds structures results were visualized and plotted by PyMOL (DeLano) and statistical analysis was performed with student's t-test by SPSS statistics 17.0 .

\section{CONFLICTS OF INTEREST}

The authors declare that they have no conflicts of interest.

\section{FUNDINGS}

This work was supported by the National Key Technologies R\&D Program for New Drugs (2014ZX09J14106-04C, 2015ZX09J15102-001), Chinese National Natural Science Foundation Projects
(81261160503) and Beijing Natural Science Foundation Project (7131012).

\section{Author contributions}

L.Z. designed research. Y.Z., C.W. and Y.C. performed experiments. Y.Z., C.W. and Y.G. analyzed the data. Y.Z., C.W. and L.Z. wrote the manuscript.

\section{REFERENCES}

1. Pagan J, Seto T, Pagano M, Cittadini A. Role of the ubiquitin proteasome system in the heart. Circulation research. 2013; 112:1046-1058.

2. Murton AJ, Constantin D, Greenhaff PL. The involvement of the ubiquitin proteasome system in human skeletal muscle remodelling and atrophy. Biochimica et biophysica acta. 2008 ; 1782:730-743.

3. Rotin D. Role of the UPS in Liddle syndrome. BMC biochemistry. 2008; 9:S5.

4. Reinstein E, Ciechanover A. Narrative review: protein degradation and human diseases: the ubiquitin connection. Annals of internal medicine. 2006; 145:676-684.

5. Dahlmann B. Role of proteasomes in disease. BMC biochemistry. 2007; 8:S3.

6. Li W, Bengtson MH, Ulbrich A, Matsuda A, Reddy VA, Orth A, Chanda SK, Batalov S, Joazeiro CA. Genome-wide and functional annotation of human E3 ubiquitin ligases identifies MULAN, a mitochondrial E3 that regulates the organelle's dynamics and signaling. PloS one. 2008; 3:e1487.

7. Deshaies RJ, Joazeiro CA. RING domain E3 ubiquitin ligases. Annual review of biochemistry. 2009; 78:399-434.

8. Rotin D, Kumar S. Physiological functions of the HECT family of ubiquitin ligases. Nature reviews Molecular cell biology. 2009; 10:398-409.

9. Hatakeyama S, Nakayama KI. U-box proteins as a new family of ubiquitin ligases. Biochemical and biophysical research communications. 2003; 302:635-645.

10. Wan L, Zou W, Gao D, Inuzuka H, Fukushima H, Berg AH, Drapp R, Shaik S, Hu D, Lester C, Eguren M, Malumbres M, Glimcher LH, Wei W. Cdh1 regulates osteoblast function through an APC/C-independent modulation of Smurf1. Molecular cell. 2011; 44:721-733.

11. Zhu H, Kavsak P, Abdollah S, Wrana JL, Thomsen GH. A SMAD ubiquitin ligase targets the BMP pathway and affects embryonic pattern formation. Nature. 1999; 400:687-693.

12. Yamashita M, Ying SX, Zhang GM, Li C, Cheng SY, Deng CX, Zhang YE. Ubiquitin ligase Smurf1 controls osteoblast activity and bone homeostasis by targeting MEKK2 for degradation. Cell. 2005; 121:101-113.

13. Kavsak P, Rasmussen RK, Causing CG, Bonni S, Zhu H, Thomsen GH, Wrana JL. Smad7 binds to Smurf2 to form 
an E3 ubiquitin ligase that targets the TGF beta receptor for degradation. Molecular cell. 2000; 6:1365-1375.

14. Aragon E, Goerner N, Zaromytidou AI, Xi Q, Escobedo A, Massague J, Macias MJ. A Smad action turnover switch operated by WW domain readers of a phosphoserine code. Genes \& development. 2011; 25:1275-1288.

15. Narimatsu M, Bose R, Pye M, Zhang L, Miller B, Ching P, Sakuma R, Luga V, Roncari L, Attisano L, Wrana JL. Regulation of planar cell polarity by Smurf ubiquitin ligases. Cell. 2009; 137:295-307.

16. Orvedahl A, Sumpter R Jr, Xiao G, Ng A, Zou Z, Tang Y, Narimatsu M, Gilpin C, Sun Q, Roth M, Forst CV, Wrana JL, Zhang YE, et al. Image-based genome-wide siRNA screen identifies selective autophagy factors. Nature. 2011; 480:113-117.

17. Moren A, Imamura T, Miyazono K, Heldin CH, Moustakas A. Degradation of the tumor suppressor Smad4 by WW and HECT domain ubiquitin ligases. The Journal of biological chemistry. 2005; 280:22115-22123.

18. Suzuki C, Murakami G, Fukuchi M, Shimanuki T, Shikauchi Y, Imamura T, Miyazono K. Smurf1 regulates the inhibitory activity of Smad7 by targeting Smad7 to the plasma membrane. The Journal of biological chemistry. 2002; 277:39919-39925

19. Zhao M, Qiao M, Oyajobi BO, Mundy GR, Chen D. E3 ubiquitin ligase Smurfl mediates core-binding factor alpha1/Runx2 degradation and plays a specific role in osteoblast differentiation. The Journal of biological chemistry. 2003; 278:27939-27944.

20. Shen R, Chen M, Wang YJ, Kaneki H, Xing L, O'Keefe R J, Chen D. Smad6 interacts with Runx2 and mediates Smad ubiquitin regulatory factor 1-induced Runx 2 degradation. The Journal of biological chemistry. 2006; 281:3569-3576.

21. Chen YL, Liu B, Zhou ZN, Hu RY, Fei C, Xie ZH, Ding $\mathrm{X}$. Smad6 inhibits the transcriptional activity of Tbx6 by mediating its degradation. The Journal of biological chemistry. 2009; 284:23481-23490.

22. Zhao L, Huang J, Guo R, Wang Y, Chen D, Xing L. Smurf1 inhibits mesenchymal stem cell proliferation and differentiation into osteoblasts through JunB degradation. Journal of bone and mineral research. 2010; 25:1246-1256.

23. Kalkan T, Iwasaki Y, Park CY, Thomsen GH. Tumor necrosis factor-receptor-associated factor-4 is a positive regulator of transforming growth factor-beta signaling that affects neural crest formation. Molecular biology of the cell. 2009; 20:3436-3450.

24. Lu K, Yin X, Weng T, Xi S, Li L, Xing G, Cheng X, Yang $X$, Zhang L, He F. Targeting WW domains linker of HECTtype ubiquitin ligase Smurf1 for activation by CKIP-1. Nature cell biology. 2008; 10:994-1002.

25. Cui Y, He S, Xing C, Lu K, Wang J, Xing G, Meng A, Jia S, He F, Zhang L. SCFFBXL(1)(5) regulates BMP signalling by directing the degradation of HECT-type ubiquitin ligase Smurf1. The EMBO journal. 2011; 30:2675-2689.

26. Cao Y, Wang C, Zhang X, Xing G, Lu K, Gu Y, He F, Zhang L. Selective small molecule compounds increase BMP-2 responsiveness by inhibiting Smurf1-mediated Smad1/5 degradation. Scientific reports. 2014; 4:4965.

27. Huang L, Kinnucan E, Wang G, Beaudenon S, Howley PM, Huibregtse JM, Pavletich NP. Structure of an E6AP-UbcH7 complex: insights into ubiquitination by the E2-E3 enzyme cascade. Science (New York, NY). 1999; 286:1321-1326.

28. Kaneki H, Guo R, Chen D, Yao Z, Schwarz EM, Zhang YE, Boyce BF, Xing L. Tumor necrosis factor promotes Runx2 degradation through up-regulation of Smurf1 and Smurf2 in osteoblasts. The Journal of biological chemistry. 2006; 281:4326-4333.

29. Guo R, Yamashita M, Zhang Q, Zhou Q, Chen D, Reynolds DG, Awad HA, Yanoso L, Zhao L, Schwarz EM, Zhang YE, Boyce BF, Xing L. Ubiquitin ligase Smurf1 mediates tumor necrosis factor-induced systemic bone loss by promoting proteasomal degradation of bone morphogenetic signaling proteins. The Journal of biological chemistry. 2008; 283:23084-23092.

30. Liu Y, Liu W, Hu C, Xue Z, Wang G, Ding B, Luo H, Tang L, Kong X, Chen X, Liu N, Ding Y, Jin Y. MiR17 modulates osteogenic differentiation through a coherent feed-forward loop in mesenchymal stem cells isolated from periodontal ligaments of patients with periodontitis. Stem cells (Dayton, Ohio). 2011; 29:1804-1816.

31. Panchenko MP, Siddiquee Z, Dombkowski DM, Alekseyev YO, Lenburg ME, Walker JD, Macgillivray TE, Preffer FI, Stone JR. Protein kinase CK1alphaLS promotes vascular cell proliferation and intimal hyperplasia. The American journal of pathology. 2010; 177:1562-1572.

32. Zhang G, Guo B, Wu H, Tang T, Zhang BT, Zheng L, He Y, Yang Z, Pan X, Chow H, To K, Li Y, Li D, et al. A delivery system targeting bone formation surfaces to facilitate RNAi-based anabolic therapy. Nature medicine. 2012; 18:307-314.

33. Fukunaga E, Inoue Y, Komiya S, Horiguchi K, Goto K, Saitoh M, Miyazawa K, Koinuma D, Hanyu A, Imamura T. Smurf2 induces ubiquitin-dependent degradation of Smurf1 to prevent migration of breast cancer cells. The Journal of biological chemistry. 2008; 283:35660-35667.

34. Melino G, Gallagher E, Aqeilan RI, Knight R, Peschiaroli A, Rossi M, Scialpi F, Malatesta M, Zocchi L, Browne G, Ciechanover A, Bernassola F. Itch: a HECT-type E3 ligase regulating immunity, skin and cancer. Cell death and differentiation. 2008; 15:1103-1112.

35. Kumar S, Tomooka Y, Noda M. Identification of a set of genes with developmentally down-regulated expression in the mouse brain. Biochemical and biophysical research communications. 1992; 185:1155-1161.

36. Shi PP, Cao XR, Sweezer EM, Kinney TS, Williams NR, Husted RF, Nair R, Weiss RM, Williamson RA, Sigmund 
CD, Snyder PM, Staub O, Stokes JB, Yang B. Salt-sensitive hypertension and cardiac hypertrophy in mice deficient in the ubiquitin ligase Nedd4-2. American journal of physiology Renal physiology. 2008; 295:F462-470.

37. Staub O, Dho S, Henry P, Correa J, Ishikawa T, McGlade J, Rotin D. WW domains of Nedd4 bind to the proline-rich PY motifs in the epithelial Na+ channel deleted in Liddle's syndrome. The EMBO journal. 1996; 15:2371-2380.

38. Kuratomi G, Komuro A, Goto K, Shinozaki M, Miyazawa K, Miyazono K, Imamura T. NEDD4-2 (neural precursor cell expressed, developmentally down-regulated 4-2) negatively regulates TGF-beta (transforming growth factorbeta) signalling by inducing ubiquitin-mediated degradation of Smad 2 and TGF-beta type I receptor. The Biochemical journal. 2005; 386:461-470.

39. Jones DC, Wein MN, Oukka M, Hofstaetter JG, Glimcher MJ, Glimcher LH. Regulation of adult bone mass by the zinc finger adapter protein Schnurri-3. Science (New York, NY). 2006; 312:1223-1227. 